\title{
3D Scanning of Semitransparent Amber with and without Inclusions
}

\author{
Adam L. Kaczmarek, Jacek Lebiedź \\ Gdansk University of Technology, Faculty of \\ Electronics, Telecommunications and \\ Informatics, ul. G. Narutowicza 11/12, 80-233 \\ Gdansk, Poland \\ adakaczm@pg.edu.pl, jacekl@eti.pg.edu.pl
}

\author{
Jakub Jaroszewicz, Wojciech Święszkowski \\ Warsaw University of Technology \\ Faculty of Materials Science and Engineering \\ ul. Wołoska 141, 02-507 Warsaw, Poland \\ jakub.jaroszewicz@pw.edu.pl \\ wojciech.swieszkowski@pw.edu.pl
}

\begin{abstract}
This paper is concerned with making 3D scans of semitransparent ambers with and without inclusions. The paper presents results of using a variety of devices applied for this purpose. Equipment used in the experiments includes a 3D laser scanner, a structured light scanner, a stereo camera, a camera array and a tomograph. The main object used in the experiment was an amber with a fossil of a lizard. The paper shows possibilities of acquiring the 3D structure of fossils embedded in semitransparent material which interfere with the measurement performed by 3D scanning equipment. Moreover, the paper shows the application of results of 3D scanning as the 3D scan of a lizard was reconstructed in a virtual reality cave making it possible to visualize in detail its shape and texture.
\end{abstract}

\section{Keywords}

3D scanning; scanning of amber; scanning of semitransparent material; VR caves

\section{INTRODUCTION}

The paper is dedicated to the problem of acquiring 3D scans of ambers. Ambers are fossils which can be semitransparent or not transparent. They can also contain inclusions such as parts of plants or insects. These fossils may have heterogeneous structure and a single amber stone may contain parts differing in their density and hue. Features of amber cause that it is particularly problematic to obtain 3D scans of this kind of specimens. One of the purposes of obtaining a 3D scan of an amber is the possibility to precisely present it to a potential purchaser. It includes both unprocessed amber stones, jewelry and other ornaments. Apart from that 3D scans of the most valuable ambers can be used to create virtual museums making it possible for virtual visitors to watch exhibits.

This paper presents the results of acquiring 3D scans with the use of different kind of scanning equipment. Authors of this paper performed experiments with scanning amber with the use of Light Detection and Ranging (LIDAR), a structured-light 3D scanner, a stereo camera, a camera array and a tomograph. Ambers scanned

Permission to make digital or hard copies of all or part of this work for personal or classroom use is granted without fee provided that copies are not made or distributed for profit or commercial advantage and that copies bear this notice and the full citation on the first page. To copy otherwise, or republish, to post on servers or to redistribute to lists, requires prior specific permission and/or a fee. in the experiments are exhibits displayed in municipal Amber Museum located in Gdansk, Poland. One of the most precious exhibits provided by the museum is amber with fossilized lizard. It is one of only few such objects in the world. Moreover, as a part of the research presented in this paper the results of scanning was used to reconstruct the fossilized lizard in virtual reality cave making it possible to precisely visualize its shape.

Original contributions of this paper includes application of a camera array for obtaining 3D scans of semitransparrent amber with inclusions, results of performing a scan using a tomograph on a fossil of an ancient lizard embeded in a amber and visualizing the result in a virtual reality cave in cooperation with a muzeum of amber. The structure of the paper is the following. Section 2 is a literature overview. Section 3 and 4 describe experiments and Section 5 presents their results. Section 6 shows the application of 3D scans to virtual reality cave. The last section presents the conclusion of the paper.

\section{RELATED WORK}

Ihrke et al. presented a detailed survey on 3D scanning of semi-transparent objects [Ihr10a]. They identified nine classes of transparency. Eight of these classes refer to levels of light transport in objects including opaque, translucent and fully transparent objects. The ninth class are inhomogeneous objects consisting of parts with various transparency. Amber can be foremost classified as an inhomogeneous object. Light passing through amber is affected by many factors including 
sub-surface scattering, reflections and absorption. Ihrke et al. described also suggestions for selecting the most suitable 3D scanning methods depending on the transparency of scanned objects. They considered performing 3D scans of objects such as glass, gas flows and fire.

Scanning transparent objects is also the subject of research of Wu et al. [Wu18a]. They developed a method for automatically and directly reconstructing complete 3D models for transparent objects based only on their appearances in a controlled environment. The environment is designed using affordable and off-the-shelf products, which include a LCD monitor, two turntables, and two cameras. This setup can work in fully automatic fashion, removing the needs for manually adjusting object positions and calibrating the cameras. The weakness of this approach is the assumption that the transparent object is homogeneous, which is rarely true in the case of amber. Interesting results were also brought by the research of the same team on the simultaneously recovering the $3 \mathrm{D}$ shape of both the wavy water surface and the moving underwater scene [Qia18a]. This approach exploits multiple viewpoints by constructing a portable camera array. However, the assumption is that the water is homogeneously transparent.

Research was also performed regarding 3D scanning of amber with inclusion. In particular, the usage of confocal laser scanning microscopy was analyzed by Clark et al. (scanning of trichomes) [Cla10a] and Zaharenko et al. (scanning of a spider) [Zak19a]. Selden took advantage of cameras, microscopes and X-ray computed tomography for imaging fossil spiders [Sel17a]. Kamp et al. showed possibilities of using X-ray microtomography with amber [Kam14a]. Li et al. analyzed amber in the context of its characteristics as a martial [Li15a].

To the best of our knowledge there were no research regarding usage of camera arrays for scanning amber with inclusions. Moreover, research papers concerned with scanning amber focus on analyzing its structure and content in the context of geological or biological research. These studies are not performed with the purpose of reconstructing amber inclusions in virtual reality cave which we present in this paper.

\section{Equipment for 3D scanning}

\section{Laser and structured-light scanners}

Laser 3D scanners produce separate clouds of points for every position of scanning. Each of these points is described by its three coordinates. They are calculated by timing the round-trip time of a pulse of light (time-of-flight laser range finder) or by analysis of the locations of the laser dots on the 2D image recorded by a camera embedded in a scanner camera related to the position of the camera and the laser emitter (triangulation). Acquiring a full specimen requires scan- ning it from all sides with some angular resolution. The created local clouds of points should then be combined into one global cloud by matching corresponding to each other distinctive points (so called reference points) identified in scanned shape for each local cloud [Kyo13a]. Then, the global cloud can be displayed directly [Lev85a, Wim06a, Dis18a] or to avoid inconsistencies it should be converted into polygon (e.g. triangle) mesh by special software.

Pure laser scanners produce only geometrical shape of object represented by a point cloud or a polygon mesh without information about color. In order to enrich the scan by color an extra camera is usually needed for assigning color for every point of the cloud or for capturing a texture applied to a larger area of the surface.

Analogically to laser scanners, structured-light 3D scanners produce also separate local clouds of points for each view of a specimen, where every point is described by three coordinates. Positions of cloud's points are calculated on the base of assessment of deformation of the multiresolutional patterns projected on the specimen and taken by an embedded camera located on the side of the projector. If the structured light is white the same camera can be used for register a color of scanned object. Otherwise, an additional camera that records the color in white light is needed. The global cloud and polygon mash are created in the same way as for laser scanners.

\section{Microcomputed tomograph}

Microcomputed tomography $(\mu \mathrm{CT})$ is a nondestructive technique that generates cross-sectional images of a sample using an X-ray source. Using these 2D representations, it is possible to generate a $3 \mathrm{D}$ reconstruction of the sample and perform analysis on the material structure. Amber with fossil inclusions is well suited for $\mu \mathrm{CT}$, both in terms of typical size and composition. The method is non-destructive and requires minimal preparation, generating 3-dimensional reconstructions that can be sectioned and viewed from numerous angles, essentially permitting digital 'dissection' of the specimen within the amber. In recent years various forms of $\mu \mathrm{CT}$ have proved particularly well-matched for imaging inclusions in amber [Die07a, Keh14a, Mor16a] and have been successfully applied in fossil lizards [Pol02a, Daz16a]. A $\mu \mathrm{CT}$ scanner looked inside the amber without damaging the fossils, allowing study researchers to digitally piece together tiny bones and examine soft tissue. The 3D images of the detailed preservation provided insight into the anatomy and ecology of ancient lizards.

Unfortunately, the tomograph is unable to register the color of the specimen. Therefore, if the geometric form itself is not satisfactory, then the reconstructed solid should be colored separately. We stopped at the noncolored reconstruction $3 \mathrm{D}$. 


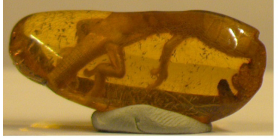

(a)

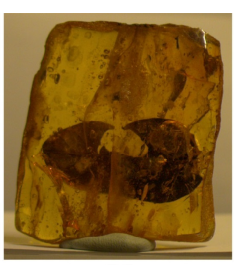

(b)

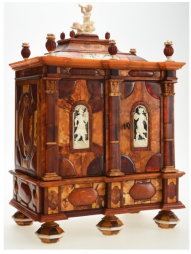

(c)
Figure 1: Amber exhibits used in experiments (a) Gierłowska's lizard (b) Amber with part of a leaf (c) Zernebach's Cabinet (photo by Michał Kosma Szczerek, courtesy of the Amber Museum, a branch of the Museum of Gdansk

\section{Stereo cameras}

A pair of cameras resembling a pair of eyes makes it possible to record $3 \mathrm{D}$ visual information of the surrounding area. Such a pair of cameras aimed in the same direction is called a stereo camera. A stereo camera produces two images taken from different points of view. Locations of the same objects is different in these images unless these objects are too distant from cameras. The difference in locations of objects in two different images is called a disparity. The closer the object is to a stereo camera, the greater is a disparity. Disparities are retrieved from a pair of images as a result of processing these images by a stereo matching algorithm which are designed to identify areas of images corresponding to the same objects.

A stereo camera consists of a reference camera and a side camera. A reference camera is a point of view of the camera set. Disparities for a series of points visible in a reference image are saved in the form of a disparity map. Disparity maps can be converted to depth maps which contain values of distances between a reference camera and objects visible in images. Converting disparity map to a depth map requires taking into account parameters of a stereo camera such as focal length of lens and the distance between constituent cameras called the baseline.

Disparity maps and depth maps obtained with the use of stereo cameras may contain errors which are incorrect values occurring in these maps. This issue is related to the selection of an appropriate stereo matching algorithm. There is a large variety of such algorithms. They differ in the quality of results, speed, the amount of required computer resources and other parameters. There are also rankings of stereo matching algorithms considering these parameters. Leading rankings of these algorithms are provided by Middlebury Stereo Vision Page [Mid21a] and the KITTI Vision Benchmark Suite [Kit21a]. The former ranks more than 100 stereo matching algorithms and the latter takes into account over 140 algorithms. Experiments presented in this paper considered well-known algorithms with available implementations. Disparity maps can also be obtained using camera arrays. [Kac17b, Kac19c] include overview of this technology.

\section{DATA ACQUISITION}

\section{Ambers used in experiments}

Amber Museum located in Gdansk, Poland provided amber fossils and items decorated with amber which were used in the experiments. Objects subjected to experiments were Baltic ambers aged about 40 million years. Some of used fossils were ambers containing inclusions. We have experimented with acquiring 3D scans of both natural amber forms with inclusions (Gierłowska's lizard - amber with lizard fossil and amber with part of a leaf) and amber works of art made by former master craftsmen (Zernebach's Cabinet).

The Gierłowska's lizard is a semitransparent Baltic amber (succinite) with inclusion of a lizard Succinilacerta succinea of the family Lacertidae. It was found by Gabriela Gierłowska (it is named after its finder) in material obtained through rinsing from Holocene fossil beach sediments about 1 kilometer away from the shore in Gdansk's Stogi district in June 1997. Unfortunately, the animal of preserved length of 3.7 centimeters is incomplete. The front part of the head, dorsal fragment of the trunk and the rearmost fragment of the tail are missing [Kos97b]. The fossil is presented in Fig. 1(a).

An amber with part of a leaf is another item used in the experiments. Similarly to the Gierłowska's lizard this object is a semitransparent Baltic amber (succinate), but it is definitely bigger $(3.5 \times 4 \mathrm{~cm})$. The specimen is shown in Fig. 1(b).

The cabinet also used in the experiments was made by a master of the Gdansk's amber guild Johann Georg Zernebach in 1724. This small box-shaped baroque piece of furniture has a shape of a cuboid with dimensions of $42 \times 31 \times 19 \mathrm{~cm}$. Its doors and drawers are made of amber, ivory, silver, mirror and wood [Kos08a]. The cabinet is presented in Fig. 1(c).

\section{Laser and structured light scanners}

Laser and structured light 3D scanners are widely used in museology. Many museum objects were archived using these devices. Although the difficulties that arise when scanning transparent and shiny objects are known, we decided to check these technologies also for amber. That is why we asked two companies dealing professionally with scanning to scan the pieces of amber presented above. One of them used a Surphaser laser scanner and a structured blue LED light ZEISS COMET L3D scanner, another one - a structured white light SmartTech Scan3D Surface scanner. The results of these tests were already described in [Leb17c], but it is worth presenting them briefly here. 


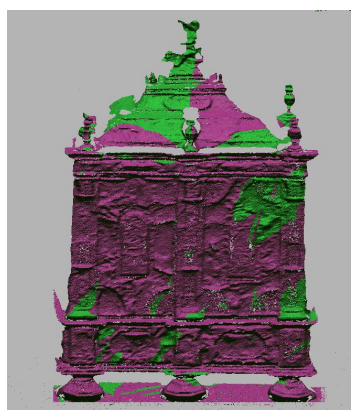

Figure 2: The laser scan of the Zernebach's Cabinet (made by Łukasz Piasecki using Surphaser scanner, courtesy of EC Test Systems) [Leb17c]

All laser 3D scanners use only a specific frequency of light. It means that process of scanning can catch only shapes visible to the used monochromatic laser light rays. The ray is reflected either from the amber exterior or from some internal amber surfaces (inclusions, fissures, structure changes etc.). If the ray enters the interior, its trajectory refracts on the external surface. For this reason, the ray measurement is disturbed. Furthermore, for complex specimens made of different kinds of amber (e.g. Zernebach's Cabinet) scanning may produce different results: a front surface for opaque fragments or a back surface (alternatively internal elements) for transparent parts [Leb17c]. This is clearly seen in Fig. 2, which presents result of use of the Surphaser laser scanner on the Zernebach's Cabinet.

Structured-light 3D scanners use rather polychromatic light (Fig. 3). Rays of a certain range of wavelength can reflect from external surfaces, while other rays can reflect from internal surfaces (inclusions, fissures, structure changes etc.). Such a diverse spectrum allow to acquire different, separate layers of amber. The heterogeneous structure of amber that has multilayer form can be reconstructed by a few correspondingly matched light rays reaching different layers of an amber and its inclusions. Unfortunately, today's scanners do not differentiate light rays relative to wavelength and this approach requires the construction of a new scanner and goes beyond the scope of described research. Existing

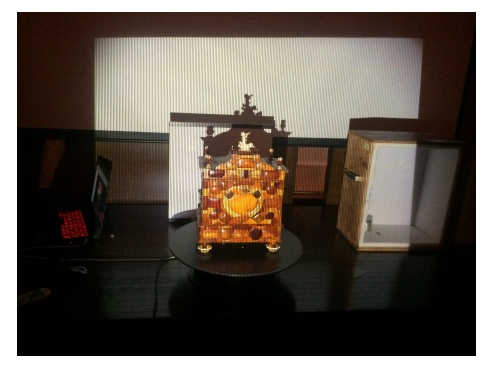

Figure 3: Process of structural light scanning of the Zernebach's Cabinet

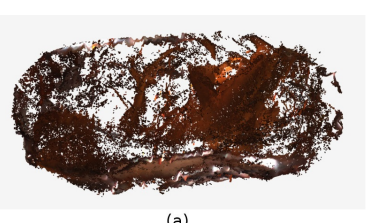

(a)

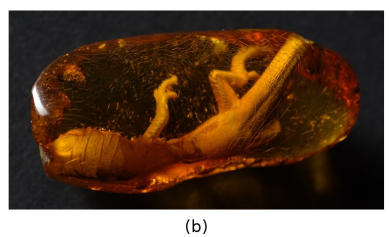

(b)
Figure 4: (a) The structured white light scan of the Gierłowska's lizard (made by Szymon Bloch using SmartTech Scan3D Surface scanner, courtesy of Scan 3D - authorized distributor of SmartTech) (b) The image corresponding to a scan (photo by Michał Kosma Szczerek, courtesy of the Amber Museum, a branch of the Historical Museum of Gdansk) [Leb17c]

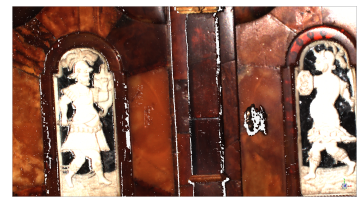

Figure 5: A structured white light scan of a central part of the Zernebach's Cabinet (made by Szymon Bloch using SmartTech Scan3D Surface scanner, courtesy of Scan 3D - authorized distributor of SmartTech) [Leb17c]

scanners can register point clouds consisting of a couple of point set layers, but they are sparse and may have a lot of holes [Leb17c], as one can see in Fig. 4 (a), which presents result of use of the SmartTech Scan3D Surface scanner based on structured white light on the Gierłowska's lizard. Fig. 4 (b) presents an image corresponding to a scan.

Analyzing results of multilayer amber scanning it is worth remembering that the reflected rays can also refract passing between different parts of an amber and its inclusions. This means that the algorithm locating individual points of the amber interior should also calculate the phenomenon of refraction. Otherwise, obtained results can be inaccurate. They will consist of a few point clouds corresponding to sequent surfaces, but reconstructed shapes inside the amber can be deformed by refraction.

The glossy ambers are an additional problem in accurate scanning both for laser and structured light scanners. These scanners cannot register surfaces where specular reflection is and point clouds have holes in these places. Therefore only matt and opaque ambers can be scanned without much difficulty, as shown in Fig. 5 presenting a structured white light scan of a central part of the Zernebach's Cabinet made by the use of the SmartTech Scan3D Surface scanner [Leb17c].

\section{Usage of tomograph}

More precise way for the 3D acquisition of amber or its inclusions is provided by X-ray tomography [Dun11a, 


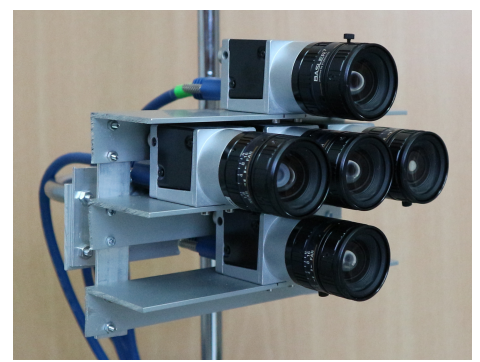

Figure 6: Real EBCA used in the experiments

Ste16a]. The basic disadvantage of this method is its huge cost but it has also some other limitations. For instance, metal parts in acquired objects make difficult to acquire amber jewelry. Fortunately, it is not a problem for natural amber forms. The size of acquired specimens is the next limitation. The dimensions of the tomographic equipment chamber limit the size of the examined object. Furthermore, the results produced by tomography are represented as memory consuming voxels instead of point clouds or easy to visualize polygon meshes generated by the other acquisition techniques.

The X-ray micro-CT observations were conducted at Warsaw University of Technology, Poland using a Zeiss Xradia XCT-400 system. Scans were performed with a polychromatic X-ray beam at an energy of $40 \mathrm{kV}$ and power of $10 \mathrm{~W}$. Sample to detector distance was set to $60 \mathrm{~mm}$, and a source to sample distance of $80 \mathrm{~mm}$ was used. Tomographic slices were generated from 1200 rotational steps through 216-degrees of rotation, using a LFOV objective, and the exposure time during each projection was set to $4 \mathrm{~s}$. Acquired images were binned $(2 \times 2 \times 2)$ giving voxel size of $38 \mu \mathrm{m}$.

\section{Usage of cameras}

Equal Baseline Camera Array (EBCA) is a device similar to a stereo camera however it produces a higher quality of disparity maps [Kac17b, Kac19c, Kac15a]. EBCA consists of a central camera and four side cameras equidistant from the central one. Side cameras are placed around a central camera forming a configuration resembling a plus sign. The image of a real EBCA used in the experiments is presented in Fig. 6. The set consists of Basler acA2500-14uc cameras with resolution $2590 \times 1942$ px and 1/2.5" sensor size.

EBCA has functions of four stereo cameras combined with each other. The considered subsets are pairs of cameras which consists of a central camera and one of side cameras. The central camera is a reference one in every such a pair. The same reference camera in every pair cause that using EBCA can be regarded as performing four measurements of the same distance with the use of four stereo cameras containing different side cameras. Perceiving EBCA in this way has many advantages and makes it possible to obtain results that have over $26 \%$ less errors than the results acquired from a single stereo camera [Kac17b].

Images from EBCA need to be analyzed in order to retrieve 3D data similarly as in case of a stereo camera. This paper presents results of using Exceptions Excluding Merging Method (EEMM) in this process [Kac17b]. This method proved to be the best one for obtaining disparity maps with the use of Equal Baseline Camera Array (EBCA). When EEMM is used disparity maps are obtained from four stereo cameras from EBCA independently from each other. Afterwards, these maps are merged together in order to acquire a resulting disparity map that has a higher quality. EEMM defines functions for processing data from four disparity maps in order to minimize the error rate in the resulting map. The method is further described in Sect. 4 of this paper.

In the experiments two stereo matching algorithms were used i.e. Semi-Global Block Matching (StereoSGBM) and Graph Cut with Expansion Moves (GC Expansion). StereoSGBM is provided with the OpenCV library [Bra08a, Hir08a]. OpenCV is a widely used open-source programming library which includes a variety of algorithms for computer vision. It also contains implementations of four stereo matching algorithms. StereoSGBM was chosen because it is the only algorithm from OpenCV classified in Middlebury ranking and it has the highest score among OpenCV algorithms in the KITTI ranking. Another stereo matching algorithm used in the experiments is Graph Cut with Expansion Moves (GC Expansion) provided by Middlebury Stereo Vision Page [Boy01a]. It is an iterative algorithm which minimizes the energy function using Markov Random Field [Sch02a]. This algorithm was selected for tests because of previous research on the five camera array used in the experiments presented in this paper [Kac17b, Kac19c]. The research showed that GC Expansion produces high quality of results when it is used with the considered array.

Images obtained with the camera set presented in Fig. 6 were used both for testing the usage of EBCA and for testing the stereo vision technology based on two cameras. Results for a stereo camera were obtained by processing an image from a central camera and an image from the left camera selected from an entire set of five images provided by EBCA. Sample images of ambers obtained by EBCA for the experiments are presented in Fig. 7.

\section{DATA PROCESSING}

\section{Processing laser and structured-light scan}

The process of combining local point clouds obtaining as a result of "one-side" static scanning into a global cloud is very complicated and so far it cannot be fully 


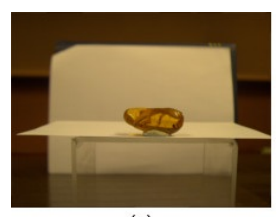

(a)

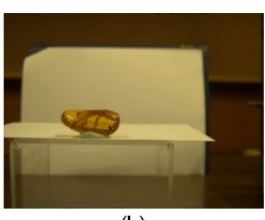

(b)

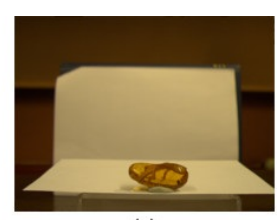

(c)

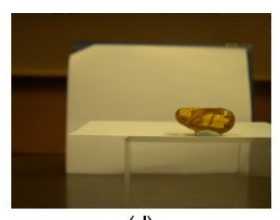

(d)

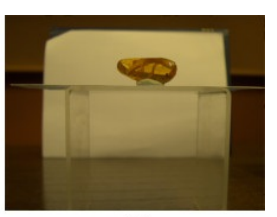

(e)

Figure 7: Images of amber with inclusion of a lizard taken with the use of (a) central, (b) right, (c) top, (d) left and (e) bottom cameras of EBCA

automated. Usually it takes the specialist more time than the process of registering points with scanner. Creating the global cloud relies on careful merging of individual local clouds. For this purpose, the corresponding reference points in combined local clouds are distinguished. Then they form the basis of combining [Kyo13a]. Setting reference points is not a trivial task. If the scanned object has many different landmark points (i.e. points in a shape that are located according to some mathematical or geometrical property) and consists of unique elements, then the choice of reference points as these landmarks is obvious. However, if the object is uniform and consists of large single-color areas with a small curvature, it is difficult to indicate the same points as reference points in aggregated scans taken from different sides. In this case, one can stick on the object special markers that will act as reference points. Unfortunately, museum curators refuse to stick such markers on their exhibits.

A separate problem is the conversion of point clouds into a polygon mesh, which is often more convenient for further processing than cloud. Fig. 8 shows point cloud of the upper part of the cabin obtained using structured blue LED light ZEISS COMET L3D scanner (a) and the triangle mesh generated by the ZEISS colin3D software (b). The number of points in such a cloud is usually very large, therefore such conversion needs simplification. The easiest method for it is decimation, consisting in leaving only every $n$-th point and omitting all other points. More sophisticated methods rely on leaving only points distinctive for a scanned shape (e.g. peaks, valleys, saddle points) [Leb17c].

In the case of transparent objects, the problem is also the separation of external surface and internal layers. Conversion of point cloud into a polygon mesh should be preceded by the separation of points into individual layers. This guarantees conversion to correct surfaces, which will be useful for the rendering mentioned as planned in Sect. 6. Automating this process is not always effective.

\section{Processing tomograph data}

Images were imported into Avizo Fire (ver. 2020.2) software platform for segmentation and 3D visualization. The powerful Watershed algorithm with interactive techniques [Rus03a] were used to provide a highly

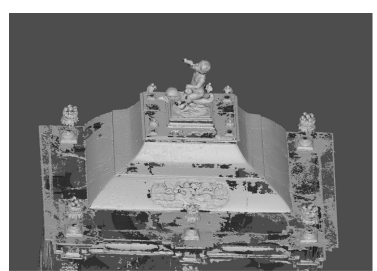

(a)

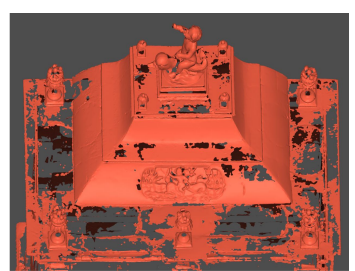

(b)
Figure 8: Visualization of the point cloud (a) and the triangle mesh (b) of the Zernebach's Cabinet (made by Łukasz Piasecki using structured blue LED light ZEISS COMET L3D scanner and the ZEISS colin3D software, courtesy of EC Test Systems)

effective strategy for segmenting complex 3D structures of the fossil lizard. The process was based on a markercontrolled algorithm which segments a gray level image based on extracted landscape image and set of markers which identify a subset of the regions of interest inside the data set. The resulting volume was manually programmed to separate the specimen from other objects embedded within the amber (mainly air bubbles). Direct Volume Rendering was used for visualizing 3D scalar fields.

\section{Processing images}

Amber fossils are visible only in parts of considered images. Therefore, only these parts were processed in order to acquire disparity maps. Remaining areas of images were not considered in calculations. The part of the image with the Gierłowska's lizard used in calculation has a size of $1000 \times 800$ pts and the size of the image of amber with a leaf was set to $1040 \times 1030$ pts.

Two stereo matching algorithms were used in experiments for obtaining disparity maps of amber. These are StereoSGBM and GC Expansion presented in Sect. 2. The obligatory input parameter of these algorithms is a range of disparities for which the algorithm verifies a match between a reference image and a side image. The selected range was wider than the real range of disparities of objects visible in images for which disparities are calculated. All other input parameters of stereo matching algorithms were set to default values or values used in examples provided with the software. 
This paper presents the comparison of disparity maps obtained using a stereo camera with disparity maps obtained using EBCA. The results for a stereo camera were based on images from the central and the right camera included in the real EBCA used in experiments. StereoSGBM and GC Expansion algorithms were used both in tests with a stereo camera and tests with five camera set.

In case of using EBCA a method of taking advantage of this five camera set needs to be applied in order to acquire disparity maps that have a higher quality than disparity map based on a single stereo camera. In the experiments presented in this paper Exceptions Excluding Merging Method (EEMM) was used for retrieving $3 \mathrm{D}$ data on the basis of five image sets [Kac17b]. The usage of EEMM consists of two steps. In the first step, stereo cameras forming EBCA are used independently from each other to obtain disparity maps. Only those stereo cameras are considered for which a central camera is a reference one. Different stereo matching algorithms designed for stereo cameras can be used in this step. Disparity maps acquired from stereo cameras are input data to the second step of Exceptions Excluding Merging Method. In the second step maps are merged in order to obtain a resulting disparity map that has a higher quality than input maps.

Each point of a disparity map contains either a value of a disparity or a value indicating that a disparity for this point is unknown. The reason for the lack of disparities is such that a stereo matching algorithm was not able to determine the disparity on the basis of images from a stereo camera that the algorithm processed. It is mainly caused by limited visibility of the same objects in two different images from a stereo camera. When EBCA consisting of five cameras is used then four disparity maps are merged into a single resulting disparity map. Therefore, every disparity in a resulting map is based on input disparities whose quantity range from 0 to 4. Exceptions Excluding Merging Method specially defines functions for merging disparities with regard to the number of input values. Details of EEMM are described in [Kac17b].

\section{RESULTS OF EXPERIMENTS}

\section{Laser and structured light 3D scanners}

Fig. 2, 5, 8 present results of scanning of the Zernebach's Cabinet by different laser and structured light scanners. Fragments of the cabinet made with opaque and matt amber were scanned with satisfactory accuracy. However, the transparent ambers have already been "lost" and the scan only showed their back wall (e.g. door corners in the Fig. 2). In the case of the Gierłowska's lizard, the scan result is a scattered cloud of points that presents both the lizard skin and amber faces (Fig. 4a). The scan result in

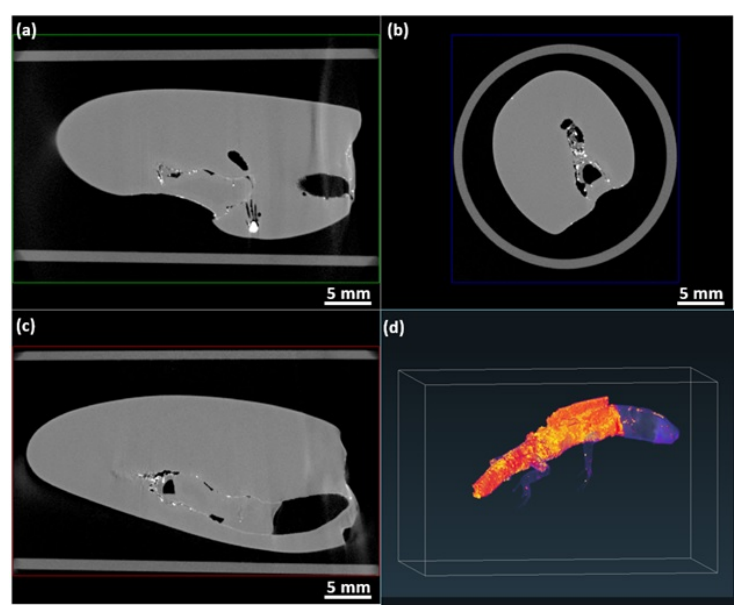

Figure 9: The $\mu \mathrm{CT}$ three perpendicular slices of the amber (a-c) and volume rendering of the Gierłowska's lizard hidden in amber

this form is unacceptable. It would require manual separation of both shapes - the lizard and the amber, which would require many hours of painstaking work of the operator. Widespread losses, especially in the cloud of points representing amber, would require manual replenishment, which in essence boils down to manual modeling of missing parts of amber shape.

\section{Tomograph}

Three perpendicular slices of the amber are shown in Fig. 9 a-c, and the whole 3D volume renderings are displayed in Fig. 9d. The extraction of 3D objects and its visualization was one of the most important steps in the analysis of the pre-processed image data. The segmentation of lizard based on gray-scale gradient was not possible for volumetric approach. The lizard could not be sufficiently separated from the surrounding structures. Even by calculating the threshold for each slice separately, we failed. Therefore, we decided to use watershed algorithm with interactive techniques [Rus03a] and finally, the 3D model was exported from special segmentation software in 3D standard triangle language (STL) format.

\section{Stereo camera and EBCA}

Fig. 10 presents disparity maps obtained in the experiments with EBCA presented in this paper. The figure has a form of a table. The first raw corresponds to results of processing images of amber with a lizard. The second row presents results for amber with part of a leaf. The column containing images (a) and (f) shows results of using the StereoSGBM algorithm for a pair of images taken by a single stereo camera. The column with images (b) and (g) presents results for the same stereo matching algorithms used with the EEMM method and a set of five images. These are the results 


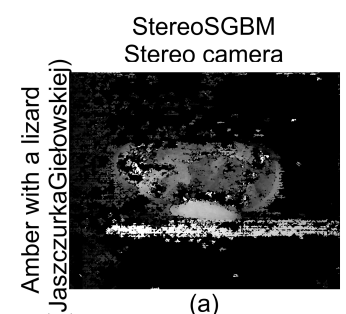

(a)

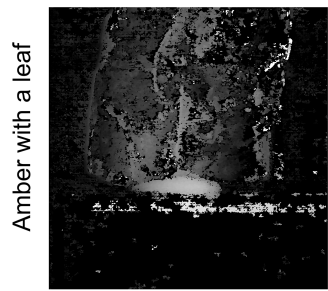

(f)

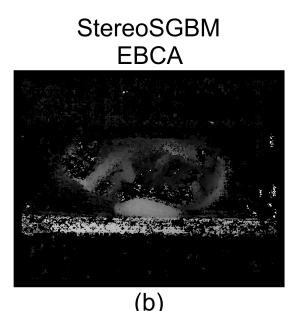

(b)

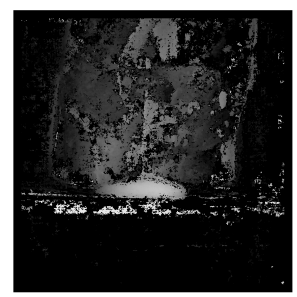

(g)

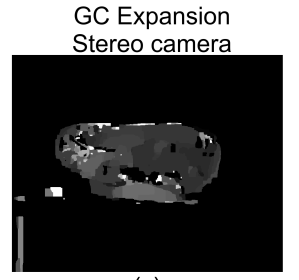

(c)

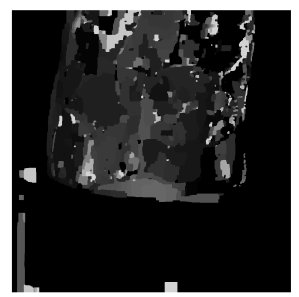

(h)

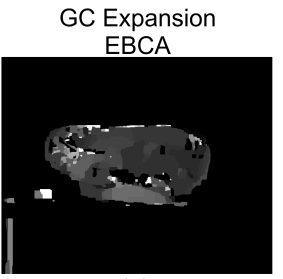

(d)

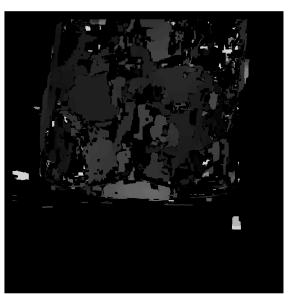

(i)

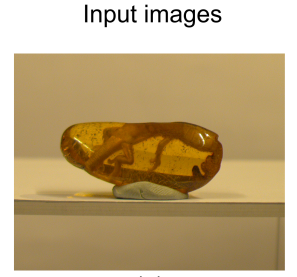

(e)

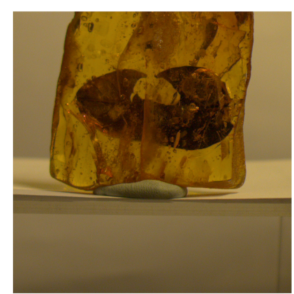

(j)

Figure 10: Results of using a stereo camera and Equal Baseline Camera Array for obtaining disparity maps of amber items

of executing the version of EEMM described in Sect. 4. Similarly, the third column presents results for the GC Expension algorithm used with a pair of images and the fourth column presents results for GC Expension applied to five image sets. The last column consists of raw images of ambers from which disparity maps were calculated.

The comparison of images presented in Fig. 10 shows differences between disparity maps obtained from two images and those obtained on the basis of five images. In both of these disparity maps there are areas with the same or similar value of a disparity depicted by a similar shade of gray in disparity maps. It can be noticed that when StereoSGBM algorithm is used edges of these areas are more irregular in case of disparity maps based on two images than in case of maps from five images. Moreover, two image disparity maps contain more parts in these areas with unknown value of disparity. These parts are more filled with disparity values in five image disparity maps. When GC Expansion is used than taking advantage of five images instead of two ones reduces the number of disparities for which the stereo matching algorithm obtained incorrect values. These disparities are visible in maps (c) and (h) as the brightest areas. Disparity maps based on five images contain only the most credible values of disparities. Instead of incorrect values of disparities these maps contain black values indicating that the disparity is unknown.

\section{3D VISUALIZATION OF ACQUIRED AMBER SPECIMENS}

Successful acquisition of 3D structure of amber specimens allows us to visualize the reconstructed objects, but the semitransparent nature of amber requires the use of advanced mathematical modeling and graphic rendering methods. A uniform 3D voxel grid, or a 3D tree such as an octree or a $k$-d tree can be used for modeling of acquired amber objects [Leb17c]. Such structures divide the object into parts and allows to define some quantities like the light transmittance for each part. Using the finite element method we can model ray tracing by solving a system of light transmittance equations corresponding to these individual parts. Such a method can simulate complex optical phenomena such as global illumination, subsurface scattering, caustics and internal reflections and offers very high quality of visualization [Leb17c].

A ray tracing engine for semitransparent materials is now being developed. It will allow the visualization of amber objects in virtual reality environments. We hope that the developing engine will be an attractive tool for curators of the Amber Museum and will allow them to prepare inviting museum exhibitions. An example of the expected capabilities of the engine is the virtual reconstruction of the Amber Room in the virtual reality cave located in the Immersive 3D Visualization Lab (Fig. 11) [Leb14a, Leb16b, Leb17c].

Precise reconstruction of the Gierłowska's lizard shape obtained thanks to the tomography allowed us to visualize the lizard itself as a zoological fossil [Leb20d]. Unfortunately, other methods do not yet provide adequate accuracy for reconstruction in VR. So we can see the three-centimeter lizard in the virtual reality cave with $100 \times$ magnification. We can walk around it and view it from any angle. Rendezvous in virtual reality with a lizard that is 44 million years old and has been enlarged to the size of a crocodile leaves an indelible impression (Fig. 12). Particularly noteworthy are the lizard's surprisingly slender fingers. 


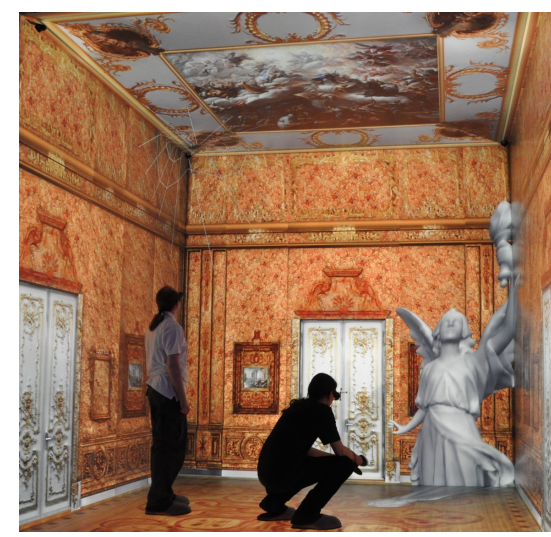

Figure 11: Visualization of the Amber Room in a virtual reality cave

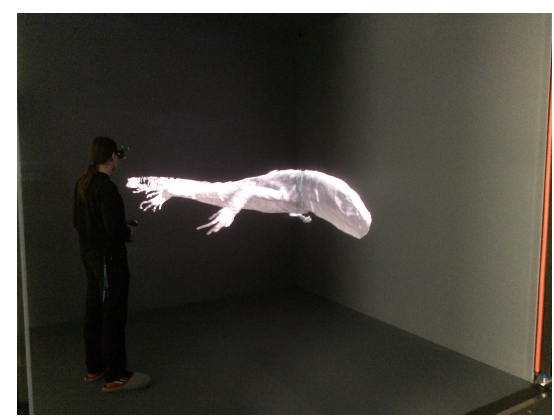

Figure 12: 3D reconstruction of the Gierłowska's lizard in a virtual reality cave with $100 \times$ magnification

\section{CONCLUSIONS}

The paper presents various classic scanning approaches applied to amber acquisition. The use of laser and structured-light scanners, microcomputed tomograph, stereo cameras and camera arrays were tested. The advantages and disadvantages of each of the approaches were described. None of the methods gave satisfactory results. The best results were obtained for tomography, but it is the most expensive and time-consuming method. In addition, it cannot cope with colors. Laser and structured light scanning as well as stereo cameras and EBCA methods capture the color correctly, but due to the translucency and multilayer structure of amber (anterior and posterior surfaces as well as inclusions and blemishes), the resulting reconstruction is burdened with significant inaccuracies. Scanning amber is a difficult task that requires further research. Amber as a structure of heterogeneous transparency, also changing abruptly (e.g. inclusions) and often with a glossy surface, seems to be one of the most difficult challenges in the acquisition of a 3D image. The conducted research has shown that it is necessary to develop special methods dedicated to amber scanning. At the moment, work is underway to develop proprietary amber scanning methods. An important element of this research is also the development of a mathematical model for the description of heterogeneity in semitransparent materials such as amber.

\section{ACKNOWLEDGMENTS}

This work was supported in part by ministry subsidies for local research and the special research infrastructure support grant 86/E-359/SPUB/SP/2019.

\section{REFERENCES}

[Boy01a] Y. Boykov, O. Veksler, R. Zabih. Fast approximate energy minimization via graph cuts. IEEE Trans. Pattern Anal. Machine Intell., 23(11):1222-1239, Nov. 2001.

[Bra08a] G. R. Bradski, A. Kaehler. Learning Opencv. First edition. O'Reilly Media, 2008.

[Cla10a] N. Clark, C. Daly. Using confocal laser scanning microscopy to image trichome inclusions in amber. J. of Paleontol. Techniques, 8:1-7, 01 2010.

[Daz16a] J. D. Daza, E. L. Stanley, P. Wagner, A. M. Bauer, D. A. Grimaldi. Mid-cretaceous amber fossils illuminate the past diversity of tropical lizards. Science Advances, 2(3), 2016.

[Die07a] M. Dierick, V. Cnudde, B. Masschaele, J. Vlassenbroeck, L. Van Hoorebeke, P. Jacobs. Micro-ct of fossils preserved in amber. Nuclear Instruments and Methods in Physics Research Section A: Accelerators, Spectrometers, Detectors and Associated Equipment, Proc. of the 10th Int. Symp. on Radiation Physics, 580(1):641-643, 2007.

[Dis18a] S. Discher, L. Masopust, S. Schulz, R. Richter, J. Döllner. A Point-Based and ImageBased Multi-Pass Rendering Technique for Visualizing Massive 3D Point Clouds in VR Environments. J. of WSCG, 26(2):76-84, 2018.

[Dun11a] J. A. Dunlop, D. Penney, N. Dalüge, P. Jäger, A. McNeil, R. S. Bradley, P. J. Withers, R. F. Preziosi. Computed tomography recovers data from historical amber: an example from huntsman spiders. Naturwissenschaften, 98(6):519527, Jun. 2011.

[Hir08a] H. Hirschmuller. Stereo processing by semiglobal matching and mutual information. IEEE Trans. Pattern Anal. Machine Intell., 30(2):328-341, Feb. 2008.

[Ihr10a] I. Ihrke, K. N. Kutulakos, H. P. A. Lensch, M. Magnor, W. Heidrich. Transparent and specular object reconstruction. Comput. Graph. Forum, 29(8):2400-2426, 2010.

[Kac15a] A. L. Kaczmarek. Improving depth maps of plants by using a set of five cameras. Journal of Electronic Imaging, 24(2):023018, 2015. 
[Kac17b] A. L. Kaczmarek. Stereo vision with equal baseline multiple camera set (ebmcs) for obtaining depth maps of plants. Computers and Electronics in Agriculture, 135:23-37, 2017.

[Kac19c] A. L. Kaczmarek. 3d vision system for a robotic arm based on equal baseline camera array. Journal of Intelligent \& Robotic Systems, 2019.

[Kam14a] T. van de Kamp, T. Rolo, T. Baumbach, L. Krogmann. Scanning the past - synchrotron Xray microtomography of fossil wasps in amber. Entomologie heute, 26:151-160, 112014.

[Keh14a] C. Kehlmaier, M. Dierick, J. Skevington. Micro-ct studies of amber inclusions reveal internal genitalic features of big-headed flies, enabling a systematic placement of metanephrocerus aczél, 1948 (insecta: Diptera: Pipunculidae). Arthropod Systematics and Phylogeny, 72:23-36, 042014.

[Kit21a] KITTI Vision Benchmark Suite, http:www.cvlibs.netdatasetskittiindex.php.

[Kos08a] B. Kosmowska-Ceranowicz. Glowing stone: Amber in polish deposits and collections. Przeglad Geologiczny (Geological Rev.), 56(8/1):604-610, 2008.

[Kos97b] B. Kosmowska-Ceranowicz, R. Kulicka, G. Gierłowska. A new find of lizard in baltic amber (in polish). Przeglad Geologiczny (Geological Rev.), 45(10):1028-1030, 1997.

[Kyo13a] T. Kyöstilä, D. C. Herrera, J. Kannala, J. Heikkilä. Merging overlapping depth maps into a nonredundant point cloud. Image Analysis, SCIA, LNCS, 7944:567-578, 2013.

[Leb14a] J. Lebiedź, A. Mazikowski. Innovative Solutions for Immersive 3D Visualization Laboratory. 22nd Int. Conf. on Computer Graphics, Vis. and Computer Vision WSCG 2014 - Comm. Papers Proc., 315-319, 2014.

[Leb16b] J. Lebiedź, J. Redlarski. Applications of immersive $3 \mathrm{~d}$ visualization lab. 24th Int. Conf. on Computer Graphics, Vis. and Computer Vision WSCG 2016 - Poster Papers Proc., CSRN 2603:69-74, 2016.

[Leb17c] J. Lebiedź, J. Redlarski, J. Rumiński. Virtual designs and reconstructions of amber works: Amber craftsman simulator. 2017 10th Int. Conf. on Human System Interactions HSI, 246-249, July 2017.

[Leb20d] J. Lebiedź. Virtual reconstruction of Gierłowska's Lizard. The Amber Magazine 44:94-97, Mar. 2020, https://www.amber.org.pl/bursztynisko.

[Lev85a] M. Levoy, T. Whitted. The use of points as a display primitive. Technical Report 85022, Computer Science Department, Univer- sity of North Carolina at Chapel Hill, Jan. 1985, https:graphics.stanford.edupaperspoints.

[Li15a] H. Li, X. Wang, Y. Zhu. Identification characteristics for amber and its imitation. Proc. of the 5th Int. Conf. on Info. Engr. for Mech. and Mtls., 483-488, 2015.

[Mid21a] Middlebury Stereo Vision Page, http:vision.middlebury.edustereo.

[Mor16a] J.-D. Moreau, D. Néraudeau, V. Perrichot, P. Tafforeau. 100-million-year-old conifer tissues from the mid-Cretaceous amber of Charente (western France) revealed by synchrotron microtomography. Ann. of Botany, 119(1):117-128, 12 2016.

[Pol02a] M. J. Polcyn, J. V. Rogers II, Y. Kobayashi, L. J. Jacobs. Computed tomography of an anolis lizard in dominican amber: Systematic taphonomic, biogeographic, and evolutionary implications. Palaeontologia Electronica, 5, 012002.

[Qia18a] Y. Qian, Y. Zheng, M. Gong, Y.-H. Yang. Simultaneous 3D Reconstruction for Water Surface and Underwater Scene. Proc. of the Eur. Conf. on Computer Vision ECCV, 776-792, 2018.

[Rus03a] J. C. Russ. The Image Processing Handbook. Fourth Edition. CRC Press 2002.

[Sel17a] P. A. Selden, D. Penney. Imaging techniques in the study of fossil spiders. Earth-Science Rev., 166:111-131, 2017.

[Sch02a] D. Scharstein, R. Szeliski. A taxonomy and evaluation of dense two-frame stereo correspondence algorithms. Int. J. of Computer Vision, 47(1):7-42, 2002. Microsoft Research Tech. Rpt. MSR-TR-2001-81, Nov. 2001.

[Ste16a] F. Stebner, R. Szadziewski, P. T. Rühr, H. Singh, J. U. Hammel, G. M. Kvifte, J. Rust. A fossil biting midge (diptera: Ceratopogonidae) from early eocene indian amber with a complex pheromone evaporator. Sc. Rpt., 6:34352, 2016.

[Wim06a] M. Wimmer, C. Scheiblauer. Instant Points: Fast Rendering of Unprocessed Point Clouds. SPBG, 129-136, July 2006.

[Wu18a] B. Wu, Y. Zhou, Y. Qian, M. Gong, H. Huang. Full 3D reconstruction of transparent objects. ACM Trans. Graph., 37(4), Article 103, Aug. 2018.

[Zak19a] A. M. Zakharenko, K. S. Golokhvast. Using confocal laser scanning microscopy to study fossil inclusion in baltic amber, a new approach. Physics and Technology of Nanostructured Mtls. IV , 806:192-196. Trans. Tech. Pub., 72019. 